\title{
Rubber hand illusion highlights massive visual capture and sensorimotor face-hand remapping in a tetraplegic man
}

\author{
Emmanuele Tidoni $^{\mathrm{a}, \mathrm{b}, *}$, Luigi Grisoni ${ }^{\mathrm{a}, \mathrm{c}}$, Marco Tullio Liuzza ${ }^{\mathrm{a}, \mathrm{b}}$ and Salvatore Maria Aglioti ${ }^{\mathrm{a}, \mathrm{b}}$ \\ ${ }^{a}$ IRCCS Fondazione Santa Lucia, Roma, Italy \\ ${ }^{\mathrm{b}}$ Dipartimento di Psicologia, Universitá di Roma La Sapienza, Roma, Italy \\ ${ }^{\mathrm{c}}$ Brain Language Laboratory, Freie Universität Berlin, Berlin, Germany
}

\begin{abstract}
.
Purpose: The illusory ownership of a fake hand as part the body follows synchronous tactile stimulation over a visible rubber hand and a covered hand. Whether brain plasticity mechanisms after sensory and motor disconnection modulates this illusion remain unexplored.

Methods: We tested a tetraplegic man after synchronous and asynchronous stimulation of the hand and face.

Results: The illusory ownership of the fake hand was tested four times in separate days and always reported. To verify whether this ownership feeling generalized also to object not resembling the human body we tested this illusion with a plastic bottle and a rubber hand. The illusionary perception of owning an external object using the rubber hand paradigm showed that the temporally matched tactile stimulation on a fake hand and visual capture mechanism create the illusionary feeling that the rubber hand was part of his body.

Conclusions: Despite lesions that dramatically disconnect the access to sensory inputs and motor outputs our data suggests a strong visual capture of a rubber hand and a possible remapping of hand-face representations after the spinal lesion. We suggest that vision and brain plasticity may represent a supportive tool for motor rehabilitation in patients with sensory deficits.
\end{abstract}

Keywords: Rubber hand illusion, visual dominance, face hand remapping, brain plasticity, spinal cord injury

\section{Introduction}

The physical self is based on the unitary perception of our body which in turn originates from visual (Lenggenhager et al., 2007), vestibular (Lopez et al., 2008 ; 2010) and proprioceptive inputs (Walsh et al., 2011; Heroux et al., 2013). A striking example of body-related multisensory integration is the so-called rubber hand illusion (RHI; Botvinick \& Cohen, 1998) in which an artificial hand may be perceived as part

*Corresponding author: Emmanuele Tidoni, Department of Psychology, Sapienza, University of Rome, Via dei Marsi 78, 00185 Roma, Italia. Tel.: +3906 49917601; Fax: +390649917635; E-mail: emmanuele.tidoni@uniroma1.it. of one's own body. The RHI is experienced when seen touches on a fake hand are delivered simultaneously to touches felt on one's own hidden hand. The RHI has both objective and subjective components. The first consists in the fact that when subjects are asked to localize their out-of-view real hand they typically localize it closer to the fake one. This is called proprioceptive drift and is considered a quantitative measure of the RHI (Longo et al., 2008). The second component is related to subjective perceptions and is explored through a questionnaire that indexes the strength of the experienced illusion. While some studies suggest objective and subjective indices are closely correlated (Longo et al., 2008) other studies suggest 
the correlation may be loose or absent (Rohde et al., 2011; Holle et al., 2011; Holmes et al., 2006). In any case, the perceived proximity to the fake hand and the subjective experience of including in one's own body an artificial part can be considered respectively a direct and indirect index of malleability of body representations. The plasticity of bodily representations is highlighted also by studies of patients with somatic and motor deafferentation. For example, people who suffered from limb amputation can experience their amputated body part still attached to their body, a sensation referred to as phantom limb (Aglioti et al., 1997; Aglioti et al. 1999; Ramachandran et al, 1998; Hunter et al., 2005; Ramachandran et al, 2010). It has recently been shown that amputee people can experience the RHI during the synchronous touch over their stump (Ehrsson et al., 2008; Schmalzl et al. 2013). Thus, the absence of a body part or the lack of its afferent/efferent connections to/from the brain may not obstacle the possibility to experience the RHI. In a similar vein, spinal cord injured (SCI) patients may experience the illusory ownership of an artificial hand. Lenggenhager et al. (2012) assessed the strength of the illusion both with questionnaire and proprioceptive drift in SCI people and found that paraplegics, as compared to the healthy controls, experienced a similar phenomenological illusion but less proprioceptive drift. Furthermore, half of the tetraplegic participants experienced both illusion and drift while the other half did not experience the illusion or the drift.

Chronic deafferentation substantially changes the functional organization of sensorimotor areas both in non-human (Chen et al., 2012) and human primates (Chen et al., 2002; Henderson et al., 2011). Perceptual correlates of functional plasticity have been reported in amputee patients who experienced the sensation of being touched on the phantom in addition to touches on specific body regions, which were not distributed randomly but reflected the known mapping of the body surface on the brain. Relevant to the present study are the studies where amputee patients refer to the phantom hand touches delivered on the face ipsilateral to the amputation side (Aglioti et al., 1997, 1999; Ramachandran et al., 2010; Pourrier et al., 2010; for a review see Nardone et al., 2013). Since hand and face are contiguously represented in the primary somatosensory cortex (SI) the above results have been interpreted as evidence that input coming from other sensory regions (e.g. face) drives the brain representation of the amputated hand. Perceptual correlates of face-hand plasticity have been reported in a patient who underwent a hand transplantation (Farnè et al., 2002). Although massive somatosensory and motor deprivation is present in both amputee and SCI people, evidence for perceptual correlates of across body part remapping in the latter group, is meager (Moore et al., 2000).

Here we studied the illusionary feeling of ownership in a spinal cord injured tetraplegic man, in whom the motor and sensory deprivation affected the hand but not the face and who has been available for extensive testing over several weeks. The aim of this study is to test whether synchronous touches applied to the face can evoke the illusory perception that the rubber hand is part of one's own body. In particular we have been able to test a young and collaborative SCI person in a modified RHI paradigm applying tactile stimulation on his real hand and face synchronously and asynchronously with the observed touches on the fake hand.

\section{Methods}

\subsection{Participant}

AP is a 30-year-old man who suffered from a traumatic spinal cord injury at C4 level (AIS level, A) seven years before his participation in the study. The completeness of the lesion was defined according to the concept of sacral sparing: sensory preservation of the perianal zone and/or motor function of the external anal sphincter (preservation of the lower sacral segments). Prior to the accident AP was mobile and independent and had no relevant medical or psychiatric history. Participant was right handed before the injury. His facial tactile sensitivity was perfectly normal and he was able to discriminate tactile stimuli over the left index finger and the left cheek. Tactile perception was tested by asking the participant to report verbally whether or not he felt Q-tip touches delivered to his, hidden-from-view, left index/cheek. The patient's facial tactile sensitivity was perfectly normal and no stimuli on the face were referred to the deafferented hand or any other body part. AP was tested in 6 experimental sessions over 12 months with a minimum temporal interval of 3 weeks. The experimental protocol was approved by the ethics committee of the Fondazione Santa Lucia and was carried out in accordance with the ethical standards of the 1964 Declaration of Helsinki. AP gave his written informed consent to take part in the study and received a reimbursement for his participation. 


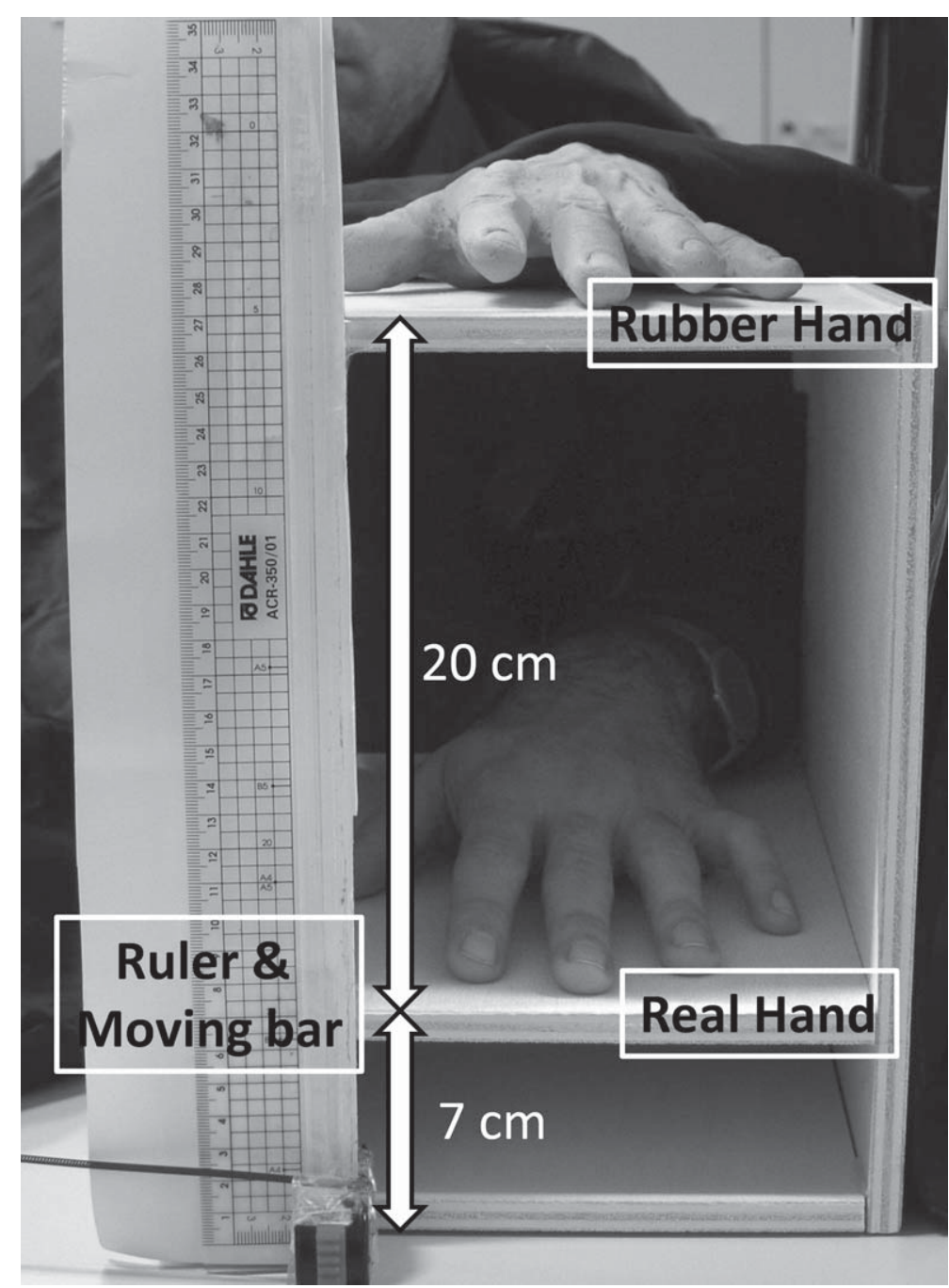

Fig. 1. Representation of the experimental apparatus.

\subsection{Procedure}

The participant was seated comfortably in his wheelchair in front of a table on which a wooden box $(27 \mathrm{~cm} \mathrm{H} \times 20 \mathrm{~cm} \mathrm{~W} \times 50 \mathrm{~cm} \mathrm{D})$ was placed. The box had two vertically distributed compartments, one for AP's left hand (lower part of the box, $7 \mathrm{~cm}$ above the table level) and one for the rubber hand (upper part of the box, $27 \mathrm{~cm}$ above the table level). The participant's hand was placed with the palm down and a left life-sized rubber hand was placed $20 \mathrm{~cm}$ higher the participant hand. AP's arm and forearm were out of his view. A black cloak covered the AP's shoulder and the proximal part of the rubber hand. In this way, the rubber hand at the top of the box was clearly visible and appeared directly connected with the AP's arm (Fig. 1).

We assessed the RHI along the vertical axis to maintain a visuo-spatial congruency between the real and the rubber hand. That is, the rubber hand was in the same location the real hand would be if the limb were lifted. In this way we maintained the real hand and the rubber hand in the same visual space to elicit maximal embodiment feelings towards the rubber hand (Bekrater-Bodmann et al., 2012). It is worth noting that with our set up the table level could not be used by AP as a visual reference for hand localization judgments. 
Table 1

Illusion related (IR) and illusion control (IC) questions after hand/face tactile stimulation conditions

\begin{tabular}{ll}
\hline & Face stimulation \\
\hline IR-1 & It seemed as if I were feeling the touch of the paintbrush at the location where I saw the rubber hand touched \\
IR-2 & It seemed as if the touch I felt was caused by the paintbrush touching the rubber hand \\
IR-3 & I felt as if the rubber hand was my own hand \\
IC-4 & I felt as if I had 2 "left hands" \\
IC-5 & I felt as if my (real) hand were drifting up (towards the rubber hand) \\
IC-6 & $\begin{array}{l}\text { It seemed as if the touch I was feeling came from somewhere } \\
\text { between my own face and the rubber hand }\end{array}$ \\
\hline
\end{tabular}

\subsection{Experiment 1}

AP was tested in 4 experimental sessions performed in non-consecutive days (minimum inter-day interval of 3 weeks). For each experimental session there were four blocks composed of a Baseline and a Stimulation phase, each lasting 3 minutes. Thus the total duration of an experimental session was of 24 minutes. The order of the blocks was counterbalanced across the different experimental sessions. In the Baseline AP simply looked at the rubber hand. In each block, the Stimulation phase always followed the Baseline. Two identical brushes were used. In particular, we stimulated the left hand and the left cheek either synchronously or asynchronously with touches on a left rubber hand that was seen by AP. In this way we had four Stimulation phases: Hand Synchronous (HS), Hand Asynchronous (HA), Face Synchronous (FS) and Face Asynchronous (FA) stimulation. In the Hand stimulation phase, the rubber and the AP's hand were stimulated on the index finger. In the face stimulation phase, the strokes were administered on the index finger of the Rubber hand and along a horizontal ideal line on the subject's cheek, starting from the muscle zygomaticus (below the left eye) towards the nose. Importantly, to avoid expectations about the timing of the tactile touches we kept an irregular temporal stroking pattern and we asked AP to wear a pair of glasses with blinkers. This prevented AP to see the brush approaching the left cheek, to be distracted by peripheral stimuli from the left side or the possibility for AP to anticipate the touch applied to his face.

Objective index of RHI. To assess any proprioceptive drift in the perceived position of the rubber hand, AP was asked to report the felt position of his hidden left index (hand localization task), by verbally stopping a sliding bar. The bar was moved in an ascending or descending way each 45 seconds by the experimenter along a ruler out of AP's sight. We counterbalanced the starting position of the bar within blocks and across the experimental sessions. This avoided AP to use any visual anchor during the localization task and to visually compare the answers between trials. Four ratings (one every 45-seconds) were obtained for each 3-minute baseline or stimulation phase, thus having 8 measures of index's perceived location for each block.

Subjective index of RHI. At the end of each stimulation phase, AP was asked to rate on a $0-10$ scale $(0=$ not at all; $10=$ very much) six statements consisting of 3 illusion-relevant (IR) and 3 illusion-control (IC) questions (see Table 1). The order of the questions was randomized in each stimulation phase.

\subsection{Results}

\subsubsection{Hand localization in the baseline condition increases over time}

We observed that AP's proprioceptive localization of his hidden hand increased every day and changed after the very first tactile stimulation. Interestingly the perceived position of his hand appeared to be at the same height of the rubber hand. We tried to support data visual inspection performing an ANOVA on the perceived hand position with Order (Base 1, Base 2, Base 3, Base 4) and Day (Day 1, Day 2, Day 3, Day 4) as between factors. Post hoc analysis was performed by means of Duncan test.

We observed a main effect of Day $\left(\mathrm{F}_{3,48}=7.31\right.$, $p<0.01, \eta^{2}=0.31$ ) with Day 1 (mean \pm s.e., $21.84 \mathrm{~cm}$ \pm 2.36 ) significantly lower relative to the other days (all $p<0.03)$ and Day $2(23.65 \mathrm{~cm} \pm 1.81)$ lower than Day 4 ( $25.65 \mathrm{~cm} \pm 0.83, p=0.02)$. All the other comparisons were not significant (all $p>0.11$ ). We found a main effect of Order $\left(\mathrm{F}_{3,48}=119.52, p<0.01, \eta^{2}=0.88\right)$ with Base $1(14.27 \mathrm{~cm} \pm 1.84)$ lower than Base 2 $(26.85 \mathrm{~cm} \pm 0.24, p<0.01)$, Base $3(26.94 \mathrm{~cm} \pm 0.16$, $p<0.01)$, and Base $4(27.38 \mathrm{~cm} \pm 0.15, p<0.01)$. Importantly the ANOVA revealed a significant double 

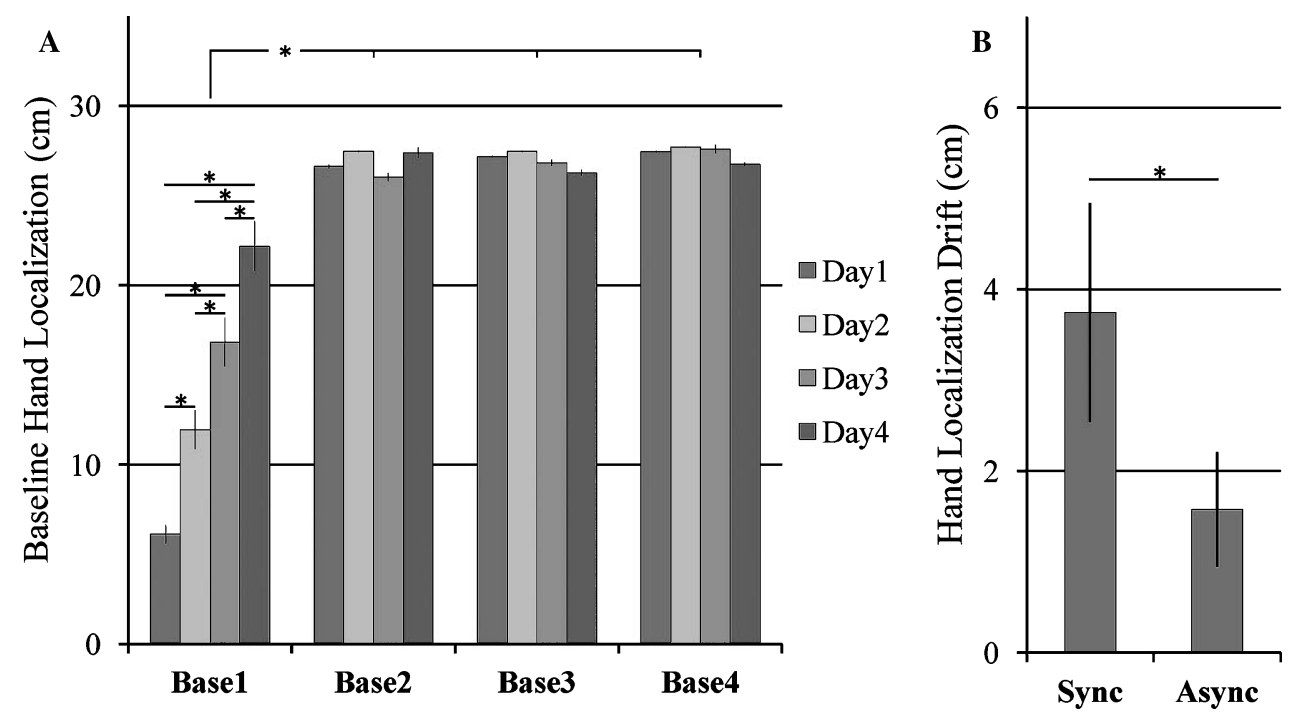

Fig. 2. Experiment 1 presentation order of Baselines for each day (A). Effect of synchronous stimulation on Proprioceptive drift (B).

interaction $\left(\mathrm{F}_{9,48}=9.19, p<0.01, \eta^{2}=0.63\right)$. Post hoc tests showed that first baselines differed each other (Base 1-Day $1<$ Base 1-Day $2<$ Base 1-Day $3<$ Base 1 -Day 4 , all $p<0.01$ ) and were lower relative to the others (all $p<0.01$; Fig. 2A).

Thus, the distance between the real and the perceived hand location increased between the experimental conditions and across the days reaching a ceiling effect after the first Baseline. Note that the mean values of Base 2-3-4 are close to the actual distance between the rubber hand and the table $(27 \mathrm{~cm})$. Importantly, we found this effect during baselines where no tactile stimulation was applied, suggesting that AP's proprioceptive judgements were largely based on vision.

\subsubsection{Proprioceptive drift}

We computed the mean for each condition (for a comprehensive view of all drift measurements see Table 3) and observed high drifts during synchronous condition $(3.74 \mathrm{~cm} \pm 1.20)$ relative to the asynchronous stimulation $(1.57 \mathrm{~cm} \pm 0.63)$. In particular the drift after face synchronous stimulation $(4.13 \mathrm{~cm} \pm 2.46)$ appeared higher than during hand stimulation $(3.36 \mathrm{~cm} \pm 2.43)$. In order to ascertain these differences and since Day and Order influenced hand localization in the Baseline phases we performed an Analysis of Covariance on proprioceptive drift (the difference between hand's localization task during Stimulation and Baseline) with Day and Order as covariates and Body-Part (Hand, Face) and
Type of Stimulation (Synchronous, Asynchronous) as between subject factors. The covariate Day was not significantly related to the participant's Proprioceptive Drift $\left(\mathrm{F}_{1,58}=1.92, p=0.17, r=-0.13, \eta^{2}=0.03\right)$ while the covariate Order was $\left(\mathrm{F}_{1,58}=40.58, p<0.01\right.$, $\left.r=-0.62, \eta^{2}=0.41\right)$. After controlling for the effect of Day and Order we found a main effect of Type of Stimulation $\left(\mathrm{F}_{1,58}=4.10, p=0.047, \eta^{2}=0.06\right.$; Fig. $\left.2 \mathrm{~B}\right)$ with higher proprioceptive drift in the Synchronous $(3.74 \mathrm{~cm} \pm 1.20)$ relative to the Asynchronous condition $(1.57 \mathrm{~cm} \pm 0.63)$. We did not find a main effect of Body Part $\left(\mathrm{F}_{1,58}=0.10, p=0.74, \eta^{2}<0.01\right)$ or interaction $\left(\mathrm{F}_{1,58}=0.15, p=0.69, \eta^{2}<0.01\right)$. These results indicate that AP's proprioceptive drift is higher during synchronous relative to asynchronous touches independently from the stimulated body part. This may reflect a face-hand remapping process that after spinal lesion facilitates the integration of tactile events applied both on the hand and on the face.

\subsubsection{Assessment of the subjective component of the RHI}

Visual inspection of IR-3 question ("I felt as if the rubber hand was my own hand") suggests high level of ownership sensation throughout all visuo-tactile manipulations. A Friedman ANOVA did not find any change over the four stimulation phases $\left(\chi_{3}=6.77\right.$, $p=0.08$; Fig. 3A). Further, mean of Illusion Relevant (IR1-3) and Illusion Control (IC4-6) questions show high scores for IR during hand stimulation, in particular 

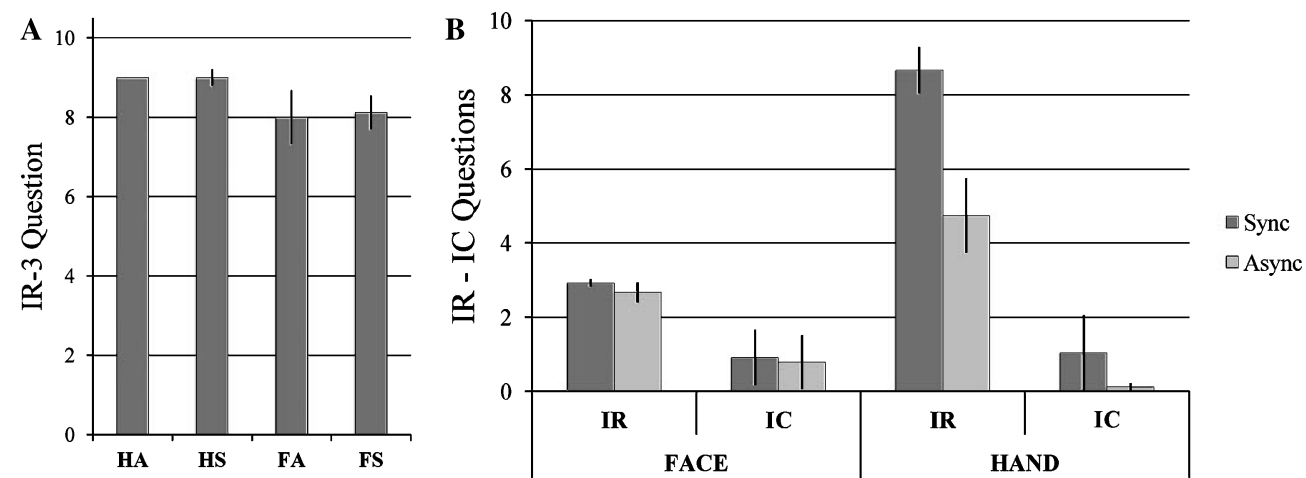

Fig. 3. Experiment 1 mean values of IR-3 for each experimental condition (A) and Illusion-Relevant (IR) and Illusion-Control (IC) mean answers in all experimental condition $(\mathrm{B})$.

Table 2

Answers to IR-2 question on a $0-10$ scale $(0=$ not at al $10=$ very much)

\begin{tabular}{|c|c|c|c|c|c|c|c|c|}
\hline & \multicolumn{2}{|c|}{ Day 1} & \multicolumn{2}{|c|}{ Day 2} & \multicolumn{2}{|c|}{ Day 3} & \multicolumn{2}{|c|}{ Day 4} \\
\hline & Async & Sync & Async & Sync & Async & Sync & Async & Sync \\
\hline HAND & 0 & 9 & 0 & 6.5 & 0.5 & 9 & 0 & $\overline{10}$ \\
\hline FACE & 0 & 0.5 & 0.1 & 0.5 & 0 & 0 & 0 & 0 \\
\hline
\end{tabular}

Table 3

Mean per condition of proprioceptive drifts of hand-face stimulation (Experiment 1) and hand-bottle stimulation (Experiment 2)

\begin{tabular}{lcccr}
\hline & Experiment 1 & & \multicolumn{2}{c}{ Experiment 2 } \\
\cline { 5 - 6 } Stimulation & mean & s.e.m. & Stimulation & mean \\
\hline HS & 3.36 & 2.43 & HS & 1.31 \\
HA & 1.61 & 1.25 & HA & 0.15 \\
FS & 4.13 & 2.46 & BS & 0.69 \\
FA & 1.54 & 1.32 & BA & 0.31 \\
\hline
\end{tabular}

higher during synchronous relative to asynchronous stimulation. Higher score for IR questions after face stimulation relative to IC but generally lower than during hand stimulation. To better understand between the visual differences in the IR and IC score we performed 3 planned comparisons (HA vs. HS; FA vs. FS; FS vs. HS) using a Wilcoxon signed rank test separately on the mean of IR and IC questions to directly test the effect of synchronicity and stimulated body part on RHI (Lenggenhager et al., 2012).

We found non-significant higher means for IRHS $(8.66 \pm 0.62)$ relative to IR-HA $(4.75 \pm 1.0$; $p=0.07$; Fig. 3B) and IR-FS (2.92 $\pm 0.1, p=0.07$, Fig. 3B). No significant difference between IR-FS and IR-FA $(2.67 \pm 0.2 ; p=0.108)$ was found. No comparisons involving IC means were significant ( $p s>0.42$; Fig. 3B).

Importantly, a qualitative inspection of answers to IR-2 question allowed us to rule out that AP's illu- sory ownership during asynchronous stimulation was driven by the inability to experience the temporal discrepancy from synchronous stimulation (see Table 2).

\subsection{Experiment 2}

In Experiment 1 AP localized his hand towards the rubber hand and reported high illusory ownership during all the blocks. Importantly we found a ceiling effect in hand localization during baselines suggesting that proprioceptive drift might be visually driven and unaffected by the stimulated body part. Thereby, to explore whether AP's visual capture of the rubber hand was specific to objects resembling the body (Tsakiris et al., 2010) we tested AP after Experiment 1 using a rubber hand and a plastic bottle.

Experiment 2 was a $2 \times 2$ design with factors Object (Rubber hand, Plastic bottle) and Type of Stimulation (Synchronous, Asynchronous) with blocks composed 

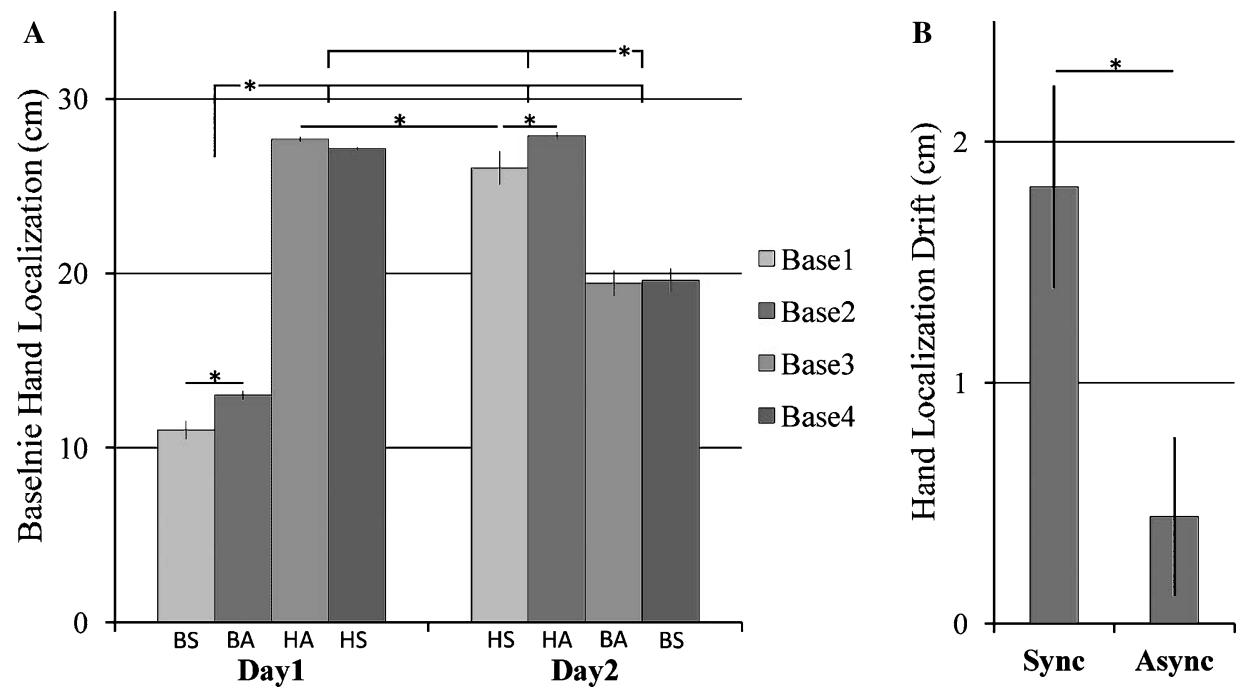

Fig. 4. Presentation order of Baselines values for each Day 1 and Day 2 (A). Effect of synchronous stimulation on Proprioceptive drift (B).

of a Baseline and a Stimulation phase as described in Experiment 1. In particular we had four stimulation phases: Hand Synchronous (HS), Hand Asynchronous (HA), Bottle Synchronous (BS) and Bottle Asynchronous (BA) stimulation. AP was tested twice and conditions' order was BS-BA-HA-HS in Day 1 and HS-HA-BA-BS in Day 2.

\subsubsection{Hand localization in the baseline condition increases over time}

We observed that AP's proprioceptive baseline localization of his hidden hand changed depending on the visually presented object. In particular it appeared that during bottle observation (Base 1-2 in Day 1, Base 3-4 in Day 2) the hand was judged to be lower $(15.65 \mathrm{~cm} \pm 1.03)$ than after the observation of a fake hand (Base 3-4 in Day 1, Base 1-2 in Day 2; $27.17 \mathrm{~cm}$ \pm 0.29 ). Known the order of the conditions we tried to support data visual inspection performing an ANOVA on perceived hand position with Order (Base 1, Base 2, Base 3, Base 4) and Day (Day 1, Day 2) as between factors. Post hoc analysis was performed by means of Duncan test.

The ANOVA revealed a Main effect of Day $\left(\mathrm{F}_{1,24}\right.$ $\left.=91.06, p<0.01, \eta^{2}=0.79\right)$ with Day $1(19.60 \mathrm{~cm}$ $\pm 2.86)$ lower than Day $2(23.22 \mathrm{~cm} \pm 1.45)$, a main effect of Order $\left(\mathrm{F}_{3,24}=41.82, p<0.01, \eta^{2}=0.84\right)$ with Base $3(23.55 \mathrm{~cm} \pm 1.60)$ and Base $4(23.35 \mathrm{~cm}$ $\pm 1.46)$ higher relative to Base $1(18.52 \mathrm{~cm} \pm 2.88$, all $p<0.01)$ and Base $2(20.23 \mathrm{~cm} \pm 2.89$, all $p<0.01)$.
Importantly we found a significant double interaction $\left(\mathrm{F}_{3,24}=307.69, p<0.01, \eta^{2}=0.97\right.$; Fig. 4A $)$. Duncan post-hoc analysis (Fig. 4A) revealed that Base 1-Day 1 $(11.02 \mathrm{~cm} \pm 0.52)$ and Base 2-Day $1(12.60 \mathrm{~cm} \pm 0.17)$ differed from one another $(p=0.04)$ and were lower than all the other baselines (all $p<0.01$ ). In contrast, Base 3-Day $2(19.42 \mathrm{~cm} \pm 0.72)$ and Base 4-Day 2 $(19.57 \mathrm{~cm} \pm 0.69)$ did not differ from each other $(p=$ 0.84 ) and were lower than all the other conditions (all $p<0.01)$. Further, Base 1-Day $2(26.02 \mathrm{~cm} \pm 0.95)$ was lower than Base 2-Day $2(27.87 \mathrm{~cm} \pm 0.19, p=0.03)$ and Base 3-Day $1(27.67 \pm 0.13, p=0.04)$.

\subsubsection{Proprioceptive drift}

We computed the mean for each condition and observed high drifts during synchronous condition $(1.81 \mathrm{~cm} \pm 0.42)$ relative to the asynchronous stimulation $(0.44 \mathrm{~cm} \pm 0.33)$. In particular the drift after bottle synchronous stimulation $(2.31 \mathrm{~cm} \pm 0.46)$ appeared higher than during hand synchronous stimulation $(1.31 \mathrm{~cm} \pm 0.69)$. In order to ascertain these differences and to control for any effect of Day and Order we performed an ANCOVA on proprioceptive drift with Day and Order as covariates and Type of Stimulation (Synchronous, Asynchronous) and Object (Hand, Bottle) as between factors. The covariate Day was significantly related to the participant's proprioceptive drift $\left(\mathrm{F}_{1,26}=9.37, p<0.01, r=0.44\right)$ while Order was not $\left(\mathrm{F}_{1,26}=0.03, p<0.86, r=-0.10\right)$. There was also a significant effect of Type of Stimulation on proprioceptive drift after controlling for the effect of Day 
Table 4

Answers to IR and IC questions in Day 1 and Day 2 after hand/bottle tactile stimulation

\begin{tabular}{|c|c|c|c|c|c|c|c|c|c|c|c|c|c|}
\hline & & \multicolumn{2}{|c|}{ IR-1 } & \multicolumn{2}{|c|}{ IR-2 } & \multicolumn{2}{|c|}{ IR-3 } & \multicolumn{2}{|c|}{ IC-4 } & \multicolumn{2}{|c|}{ IC-5 } & \multicolumn{2}{|c|}{ IC-6 } \\
\hline & & Async & Sync & Async & Sync & Async & Sync & Async & Sync & Async & Sync & Async & Sync \\
\hline \multirow[t]{2}{*}{ Day 1} & Bottle & 0 & 10 & 0 & 0 & 0 & 0 & 0 & 0 & 0 & 0 & 0 & 5 \\
\hline & Hand & 0 & 10 & 0 & 8 & 9 & 9 & 0 & 0 & 0 & 0 & 0 & 0 \\
\hline \multirow[t]{2}{*}{ Day 2} & Bottle & 0 & 8 & 1 & 8 & 1 & 6 & 0 & 1 & 0 & 1 & 0 & 1 \\
\hline & Hand & 0 & 9 & 2 & 9.5 & 9 & 9 & 0 & 1 & 0 & 0 & 1 & 0 \\
\hline
\end{tabular}

$\left(\mathrm{F}_{1,26}=7.75, p<0.01\right.$; Fig. 4B) with higher drift in the Synchronous condition $(1.81 \mathrm{~cm} \pm 0.42)$ relative to the Asynchronous condition $(0.44 \pm 0.33)$. No main effect of Object $\left(\mathrm{F}_{1,26}=2.83, p=0.10\right)$ or interaction $\left(\mathrm{F}_{1,26}=0.21, p=0.64\right)$ was found.

\subsubsection{Illusion subjective assessment}

Subjective reports were collected twice for each stimulation phase. A qualitative inspection of Table 4 suggests that IR questions were higher for the HS relative to the other conditions and that IR-3 was similar for $\mathrm{HA}$ and $\mathrm{HS}$ and higher relative to $\mathrm{BS}$ and $\mathrm{BA}$ conditions.

Further in Day 2, AP experienced a slight illusory ownership of the bottle. Similar findings have been described in healthy people who experienced level of ownership towards a table during synchronous but not asynchronous tactile stimulation (Armel \& Ramachandran, 2003). The fact that AP's illusory ownership of a bottle differed between the two days may hint at a higher malleability and variability of body representation during synchronous stimulation, and at a lower (although non null) degree of embodiment of external objects not resembling body parts.

\section{Discussion}

By using a modified version of the RHI paradigm we extensively tested a young and collaborative tetraplegic man with the aim of exploring two main issues: i) the possible massive effect of vision of a deafferented body part in modulating both subjective and objective components of the illusion of embodying an artificial hand; ii) the possible effects of tactile stimuli delivered to non-deafferented body parts (but represented contiguously in the somatosensory system downstream the lesion level, e.g. the face) in modulating the RHI. Two novel findings were obtained. The first is that in conditions of massive deafferentation, an overwhelming visual capture effect, specific for an artificial hand and not extending to non-bodily objects, may help to represent deafferented body parts. The second is that delivering tactile stimuli on the hemiface ipsilateral to the deafferented hand increases the sense of embodiment as inferred from RHI and thus hints at the presence of a plastic remapping of face-hand representations.

\subsection{RHI and visual capture}

In Experiment 1, using a modified RHI paradigm (Botvinick and Cohen, 1998), we applied synchronous and asynchronous tactile stimulation to the left hand and cheek while the subject was looking at a rubber hand. We assessed both subjective and objective measures of ownership by means of questionnaire and proprioceptive drift. To control for the influence of vision on hand localization after tactile stimulation the tetraplegic patient was asked to watch the rubber hand and indicate the position of his hidden real hand every 45 seconds for 4 times. During baseline sessions AP looked at the rubber hand without any tactile stimulation applied to his hand or face. AP showed high level of ownership of the rubber hand in all the experimental manipulations as measured by questionnaire and high proprioceptive drift during synchronous stimulation. Further in Experiment 2 we tested whether AP visual capture was limited to objects resembling body parts (rubber hand) or could be affected also by non-body related objects (plastic bottle). We observed an effect of synchronous stimulation on proprioceptive drift and ownership during the observation of the rubber hand.

Overall our data suggest that the participant experienced the rubber hand as his own and located his real hand towards the artificial hand likely through a visual capture of external objects resembling the human body over proprioception.

\subsection{Face-hand remapping and visual capture}

Our data show that proprioceptive drift is higher whenever hand and face are touched synchronously 
with the rubber hand and AP's experience of owning the artificial hand is stable throughout all experimental conditions. Thus face-hand remapping mechanism and vision differently modulated the proprioceptive localization of the hidden hand and the subjective ownership of an external object.

Previous RHI studies in patients revealed that intact integration processes of visual and tactile information are relevant for proprioceptive drift and illusory ownership. Dystonic patients (Fiorio et al., 2011) and SCI people (Lenggenhager et al., 2012), for example, showed absence of proprioceptive drift during synchronous visuo-tactile stimulation compared to healthy participants.

Contrary to these findings, data suggests AP has preserved facilitation of multisensory integration for both hand and face and he strongly relies on visual information to experience a stable illusory ownership of the rubber hand. These results are in keeping with what found in patients with complex regional pain syndrome who, despite their impairment in processing static tactile stimuli, show preserved ability to integrate multisensory events and to perceive illusory ownership of a fake hand (Reinersmann et al., 2013). Further, a recent study on two SCI people reported a recovery of tactile sensitivity after RHI suggesting a strong reliance on visual information to overcome the absence of proprioceptive inputs (Lenggenhager et al., 2013). Interestingly, plastic interactions between face and hand cortical representation occurs in patients with facial paralysis. Haenzi and colleagues (2013) revealed that tactile stimulation to the hand activates the cortical representation of the face in people who had undergone cosmetic injections of botulinum toxin to treat wrinkles.

To the best of our knowledge this is the first study to report an experience of ownership toward an external object in a tetraplegic person during face and hand tactile stimulation. The suggested strong reliance on visual information for body ownership may erroneously be considered as limited to people with proprioceptive and sensorimotor deficit. Indeed, the observation in a mirror box of the reflected opposite arm elicits the sensation in the amputee patient that their phantom arm is moving (Ramachandran et al., 1995) and induce involuntary movements in normal participants (Romano et al., 2013). However healthy subjects during high level of full body ownership reported asynchronous touches as congruent (Maselli and Slater, 2013). Moreover a phantom arm (Guter- stam et al., 2013) as well as a telescoped limb illusion (Schmalzl \& Ehrsson, 2011) can be induced using the rubber hand paradigm. Finally individuals with visiontouch synesthesia experienced proprioceptive drift and ownership towards a rubber hand in the absence of touch on their real hands (Aimola Davies et al., 2013) and a recent study showed that touch expectation modulates the illusory sensation of ownership towards the rubber hand (Ferri et al., 2013). It is worth noting that, when at the end of some experimental blocks we intentionally rotated the rubber hand of $90^{\circ}$ (like in Tsakiris \& Haggard, 2005) or $180^{\circ}$ (like in Schmalzl et al., 2013; Petkova \& Ehrsson, 2009; Guterstam et al., 2011), AP immediately reported the loss of owning the fake hand. On the contrary, mere observation of a the fake hand positioned in anatomically congruent position, induced in AP the strong feeling that the artificial hand was part of his body. Such feeling disappeared when the rubber hand was rotated so as to be in an anatomical implausible position. Thus, the strong effect of vision on embodiment takes into account the anatomical topography of the body ruling out that any response bias played a role in the results. This is reminiscent of the result obtained in a population of anosognosic patients who reported the illusory ownership of a rubber hand only when placed in front of them according to a humanly plausible posture (Fotopoulou et al., 2008). In a similar vein, embodiment of the hand of an experimenter by simply watching it has been reported in a sub-group of patients with anosognosia for hemiplegia (Garbarini et al., 2013). Overall these studies and our results indicate that visual information may play a crucial role in modulating the sense of body ownership particularly in people with sensorimotor deficits.

Unlike the classic RHI paradigm where proprioceptive assessment of baseline is measured with the rubber hand out of participant's view (Botvinick \& Cohen, 1998; Tsakiris et al., 2010; Petkova et al., 2012), our baseline was characterized by mere observation of the rubber hand. This procedure allowed us to assess the role of vision on proprioceptive judgments before each stimulation phase. The extracted proprioceptive drift thus represents a vision-unbiased effect of visuo-tactile processing following hand and face stimulation. We found an overwhelming visual dominance of the fake hand over proprioception and ownership feeling.

Importantly only synchronous stimulation further increased the proprioceptive drift suggesting that sensory information from face and hand are similarly processed. An excessive observation of the rubber hand 
cannot explain the baseline's ceiling effect in the third experimental condition of each day. Indeed at the end of the second phase AP's hand was moved, the cloak was removed, the rubber hand was hidden (or momentarily rotated) and the third phase started after a pause of nearly 10 minutes. Further our data cannot be attributed to the inability of the patient to correctly categorize synchronous and asynchronous stimulations. As a matter of fact, AP was able to tell apart synchronous from asynchronous stimulation as highlighted by his answers to IR-2 question (see Table 3). We reported both descriptive and inferential analysis. Although the inferential approach on single cases may inflate error Type I probability, this procedure has been used in many single case reports (Pia et al., 2013; Garbarini et al., 2012; Benson et al., 2012). By performing an ANCOVA we aimed at partialling out the variance explained by DAY and ORDER. This allowed us to find an effect of synchronicity irrespective of the stimulated body part (i.e. face, hand).

Thus our data may indicate that vision drives both illusion and proprioception during synchronous tactile stimulation on hand and that face stimulation additionally increases the proprioceptive drift towards the observed artificial hand.

\section{Conclusion}

RHI may represent an alternative and new tool to support motor rehabilitation (Dietz et al., 2013) in SCI people (Lenggenhager et al., 2013) suggesting that exploring illusory ownership may be relevant for the clinics and the rehabilitation medicine. Importantly this modified RHI paradigm may represent an indirect way to test brain plasticity and body representation in SCI people who experience a dramatic change in body perception and visual representation (Fuentes et al., 2013). Studies on larger samples may allow to support more robust conclusions on possible hand-face remapping mechanisms after spinal cord lesion (Scandola et al., 2013; Scandola et al., 2014) and therefore to understand the role of across-body parts remapping in supporting the sensory side of motor rehabilitation (Lenggenhager et al., 2013; Dietz et al., 2013) as well as the reduction of neuropathic pain (e.g. using virtual reality, Villiger et al., 2011).

Finally these results suggest that alterations of somatosensory inputs and motor outputs as well as the disconnection of the body from the brain due to spinal cord lesions may result in a disturbed sense of an embodied self. Furthermore, plasticity-related cortical changes might influence the dynamics of the bodily representation and may help understanding cortical remapping and the development of novel rehabilitation procedures for people with reduced access to sensorimotor information.

\section{Acknowledgments}

Financial support from the EU Information and Communication Technologies Grant (VERE project, FP7-ICT-2009-5, Prot. Num. 257695), the Italian Ministry of Health (and RF-2010-2312912). We thank AP for his help and useful suggestions, Bigna Lenggenhagger for her comments on the initial stage of the study and Gabriele Fusco for his help in collecting data.

\section{References}

Aglioti, S., Smania, N., Atzei, A., \& Berlucchi, G. (1997). Spatiotemporal properties of the pattern of evoked phantom sensations in a left index amputee patient. Behav Neurosci, 111(5), 867872.

Aglioti, S.M., Beltramello, A., Peru, A., Smania, N., \& Tinazzi, M. (1999). Anomalous double sensations after damage to the cortical somatosensory representation of the hand in humans. Neurocase, 5(4), 285-292.

Aimola Davies, A.M., \& White, R.C. (2013). A sensational illusion: Vision-touch synaesthesia and the rubber hand paradigm. Cortex, 49(3), 806-818.

Armel, K.C., \& Ramachandran, V.S. (2003). Projecting sensations to external objects: Evidence from skin conductance response. Proc Biol Sci, 270(1523), 1499-1506.

Bekrater-Bodmann, R., Foell, J., Diers, M., \& Flor, H. (2012). The perceptual and neuronal stability of the rubber hand illusion across contexts and over time. Brain Res, 1452, 130-139.

Benson, V., Ietswaart, M., \& Milner, D., (2012). Eye Movements and Verbal Report in a Single Case of Visual Neglect. PloS One, 7 , e43743.

Botvinick, M., \& Cohen, J. (1998). Rubber hands "feel" touch that eyes see. Nature, 391(6669), 756.

Chen, L.M., Qi, H.X., \& Kaas, J.H. (2012). Dynamic reorganization of digit representations in somatosensory cortex of nonhuman primates after spinal cord injury. J Neurosci, 32(42), 1464914663.

Chen, R., Cohen, L.G., \& Hallett, M. (2002). Nervous system reorganization following injury. Neuroscience, 111(4), 761-773.

Dietz, V., \& Fouad, K. (2013). Restoration of sensorimotor functions after spinal cord injury. Brain, 137, 654-667.

Ehrsson, H.H., Rosén, B., Stockselius, A., Ragnö, C., Köhler, P., \& Lundborg, G. (2008). Upper limb amputees can be induced to experience a rubber hand as their own. Brain, 131, 3443-3452. 
Farnè, A., Roy, A.C., Giraux, P., Dubernard, J.M., \& Sirigu, A. (2002). Face or hand, not both: Perceptual correlates of reafferentation in a former amputee. Curr Biol, 12, 13421346.

Ferri, F., Chiarelli, A.M., Merla, A., Gallese, V., \& Costantini, M. (2013). The body beyond the body: Expectation of a sensory event is enough to induce ownership over a fake hand. Proc Biol Sci, 280(1765), 20131140.

Fiorio, M., Weise, D., Önal-Hartmann, C., Zeller, D., Tinazzi, M., \& Classen, J. (2011). Impairment of the rubber hand illusion in focal hand dystonia. Brain, 134, 1428-1437.

Fotopoulou, A., Tsakiris, M., Haggard, P., Vagopoulou, A., Rudd, A., \& Kopelman, M. (2008). The role of motor intention in motor awareness: An experimental study on anosognosia for hemiplegia. Brain, 131, 3432-3442.

Fuentes, C.T., Pazzaglia, M., Longo, M.R., Scivoletto, G., \& Haggard, P. (2013). Body image distortions following spinal cord injury. J Neurol Neurosurg Psychiatry, 84(2), 201-207.

Garbarini, F., Rabuffetti, M., Piedimonte, A., Pia, L., Ferrarin, M., Frassinetti, F., Gindri, P., Cantagallo, A., Driver, J., \& Berti, A. (2012). "Moving" a Paralysed Hand: Bimanual Coupling Effect in Patients with Anosognosia for Hemiplegia. Brain, 135, 1486-1497.

Garbarini, F., Pia, L., Piedimonte, A., Rabuffetti, M., Gindri, P., \& Berti, A. (2013). Embodiment of an alien hand interferes with intact-hand movements. Curr Biol, 23(2), R57-R58.

Guterstam, A., Gentile, G., \& Ehrsson, H.H. (2013). The invisible hand illusion: Multisensory integration leads to the embodiment of a discrete volume of empty space. J Cogn Neurosci, 25(7), 1078-1099.

Guterstam, A., Petkova, V.I., \& Ehrsson, H.H. (2011). The illusion of owning a third arm. PloS one, 6(2), e17208.

Haenzi, S., Stefanics, G., Lanaras, T., Calcagni, M., \& Ghosh, A. (2013). Altered cortical activation from the hand after facial botulinum toxin treatment. Ann Clin Transl Neurol, 1, 6468.

Henderson, L.A., Gustin, S.M., Macey, P.M., Wrigley, P.J., \& Siddall, P.J. (2011). Functional reorganization of the brain in humans following spinal cord injury: Evidence for underlying changes in cortical anatomy. J Neurosci, 31(7), 2630-2637.

Héroux, M.E., Walsh, L.D., Butler, A.A., \& Gandevia, S.C. (2013). Is this my finger? Proprioceptive illusions of body ownership and representation. J Physiol, 591, 5661-5670.

Holle, H., McLatchie, N., Maurer, S., \& Ward, J. (2011). Proprioceptive drift without illusions of ownership for rotated hands in the "rubber hand illusion" paradigm. Cogn Neurosci, 2(3-4), 171-178.

Holmes, N.P., Snijders, H.J., \& Spence, C. (2006). Reaching with alien limbs: Visual exposure to prosthetic hands in a mirror biases proprioception without accompanying illusions of ownership. Percept Psychophys, 68(4), 685-701.

Hunter, J.P., Katz, J., \& Davis, K.D. (2005). Dissociation of phantom limb phenomena from stump tactile spatial acuity and sensory thresholds. Brain, 128, 308-320.

Lenggenhager, B., Tadi, T., Metzinger, T., \& Blanke, O. (2007). Video ergo sum: Manipulating bodily self-consciousness. Science, 317(5841), 1096-1099.
Lenggenhager, B., Pazzaglia, M., Scivoletto, G., Molinari, M., \& Aglioti, S.M. (2012). The sense of the body in individuals with spinal cord injury. PloS one, 7(11), e50757.

Lenggenhager, B., Scivoletto, G., Molinari, M., \& Pazzaglia, M. (2013). Restoring tactile awareness through the rubber hand illusion in cervical spinal cord injury. Neurorehabil Neural Repair, 27(8), 704-708.

Longo, M.R., Schüür, F., Kammers, M.P.M., Tsakiris, M., \& Haggard, P. (2008). What is embodiment? A psychometric approach. Cognition, 107(3), 978-998.

Lopez, C., Halje, P., \& Blanke, O. (2008). Body ownership and embodiment: Vestibular and multisensory mechanisms. Clin Neurophysiol, 38(3), 149-161.

Lopez, C., Lenggenhager, B., \& Blanke, O. (2010). How vestibular stimulation interacts with illusory hand ownership. Conscious Cogn, 19(1), 33-47.

Maselli, A., \& Slater, M. (2013). The building blocks of the full body ownership illusion. Front Hum Neurosci, 7, 83.

Moore, C.I., Stern, C.E., Dunbar, C., Kostyk, S.K., Gehi, A., \& Corkin, S. (2000). Referred phantom sensations and cortical reorganization after spinal cord injury in humans. Proc Natl Acad Sci USA, 97(26), 14703-14708.

Nardone, R., Höller, Y., Brigo, F., et al. (2013). Functional brain reorganization after spinal cord injury: Systematic review of animal and human studies. Brain Res, 1504, 58-73.

Petkova, V.I., \& Ehrsson, H.H. (2009). When right feels left: Referral of touch and ownership between the hands. PloS one, 4(9), e6933.

Petkova, V.I., Zetterberg, H., \& Ehrsson, H.H. (2012). Rubber hands feel touch, but not in blind individuals. PloS one, 7(4), e35912.

Pia, L., Spinazzola, L., Rabuffetti, M., Ferrarin, M., Garbarini, F., Piedimonte, A., Driver, J., \& Berti A. (2013) Temporal coupling due to illusory movements in bimanual actions: Evidence from anosognosia for hemiplegia. Cortex, 49, 16941703.

Pourrier, S.D., Nieuwstraten, W., Van Cranenburgh, B., Schreuders, T.A.R., Stam, H.J., \& Selles, R.W. (2010). Three cases of referred sensation in traumatic nerve injury of the hand: Implications for understanding central nervous system reorganization. J Rehabil Med, 42(4), 357-361.

Ramachandran, V.S., Rogers-Ramachandran, D., \& Cobb, S. (1995). Touching the phantom limb. Nature, 377, 489-490.

Ramachandran, V. (1998). The perception of phantom limbs. The D. O. Hebb lecture. Brain, 121(9), 1603-1630.

Ramachandran, V.S., Brang, D., \& McGeoch, P.D. (2010). Dynamic reorganization of referred sensations by movements of phantom limbs. Neuroreport, 21(10), 727-730.

Reinersmann, A., Landwehrt, J., Krumova, E.K., Peterburs, J., Ocklenburg, S., Güntürkün, O., \& Maier, C. (2013). The rubber hand illusion in complex regional pain syndrome: Preserved ability to integrate a rubber hand indicates intact multisensory integration. Pain, 154(9), 1519-1527.

Rohde, M., Di Luca, M., \& Ernst, M.O. (2011). The Rubber Hand Illusion: Feeling of ownership and proprioceptive drift do not go hand in hand. PloS one, 6(6), e21659. 
Romano, D., Bottini, G. \& Maravita, A., (2013). Perceptual effects of the mirror box training in normal subjects. Restor Neurol Neurosci, 31(4), 373-386.

Scandola M., Tidoni E., Grisoni L., Moro V., \& Aglioti S.M. (2013). The Rubber Hand Illusion after face stimulation in Spinal Cord Injured people. Aegina Summer School 2013 - Embodied Intersubjectivity: The 1st person and the 2 nd person perspective Aegina, Greece.

Scandola, M., Tidoni, E., Avesani, R., Brunelli, G., Aglioti, S. M., \& Moro, V. (2014). Rubber hand illusion induced by touching the face ipsilaterally to a deprived hand: Evidence for plastic "somatotopic" remapping in tetraplegics. Frontiers in Human Neuroscience, 8, 404

Schmalzl, L., \& Ehrsson, H.H. (2011). Experimental induction of a perceived "telescoped" limb using a full-body illusion. Front Hum Neurosci, 5, 34

Schmalzl, L., Kalckert, A., Ragnö, C., \& Ehrsson, H.H. (2013). Neural correlates of the rubber hand illusion in amputees: A report of two cases. Neurocase, 37-41.
Tsakiris, M., \& Haggard, P. (2005). The rubber hand illusion revisited: Visuotactile integration and self-attribution. J Exp Psychol Hum Percept Perform, 31, 80-91.

Tsakiris, M., Carpenter, L., James, D., \& Fotopoulou, A. (2010). Hands only illusion: Multisensory integration elicits sense of ownership for body parts but not for non-corporeal objects. Exp brain Res, 204(3), 343-352.

Villiger, M., Estévez, N., Hepp-Reymond, M.-C., Kiper, D., Kollias, S.S., Eng, K., \& Hotz-Boendermaker, S. (2013). Enhanced activation of motor execution networks using action observation combined with imagination of lower limb movements. PloS one, 8(8), e72403.

Walsh, L.D., Moseley, G.L., Taylor, J.L., \& Gandevia, S.C. (2011) Proprioceptive signals contribute to the sense of body ownership. J Physiol, 589, 3009-3021. 